\title{
Biofilia: produção de vida ativa em cuidados paliativos
}

\author{
Biophilia: production of active life in palliative care
}

\author{
Amanda Amorim Zanatta1, Robiran José Santos-Junior' ${ }^{\mathbf{1}}$ Carla Corradi Perini1, Marta Luciane \\ Fischer $^{\mathbf{1}}$
}

DOI: 10.1590/0103-1104201912223

RESUMO Considerando os benefícios biopsicossociais decorrentes da interação humanos/ natureza, questionou-se como a hipótese da biofilia tem sido inserida nos cuidados paliativos. Objetivou-se mapear, através de revisão integrativa, a concepção histórica e científica da temática, atestando a contemplação dos princípios dos cuidados paliativos. De 1.928 textos científicos, 71 abarcaram o tema, sendo possível identificar, ao menos, um dos nove princípios, com destaque para a integração com aspectos psicológicos e espirituais. A biofilia foi aplicada, sobretudo, ao aspecto emocional, e a zooterapia, ao relacional. Contudo, diante das limitações impostas ao atendimento de aspectos de biossegurança e dependência do voluntariado, propõe-se a inserção da horticultura terapêutica em hospices e visita do animal de estimação.

PALAVRAS-CHAVE Bioética. Cuidados paliativos. Horticultura terapêutica. Terapia assistida por animais. Vulnerabilidade em saúde.

ABSTRACT Considering the biopsychosocial benefits derived from human/nature interaction, questions were raised considering how the biophilia theory has been inserted in palliative care. The objective was to map, through an integrative review, the historical and scientific conception of the subject, attesting the contemplation of the principles of palliative care. From 1.928 scientific texts, 71 covered the theme, being possible to identify, at least, one of the nine principles, with special focus on integration with psychological and spiritual aspects. The biophilia was applied, mainly, to the emotional aspect, and the zootherapy, to the relational aspect. However, in view of the limitations imposed on attending to aspects of biosafety and reliance on volunteering, it is proposed the insertion of therapeutic horticulture in hospices and visit of the pet.

KEYWORDS Bioethics. Palliative care. Horticultural therapy. Animal assisted therapy. Health vulnerability. 


\section{Introdução}

As relações estabelecidas entre os seres humanos e a natureza fomentaram alterações significativas na percepção e representação dos elementos naturais ao longo do seu percurso evolutivo. As relações, inicialmente instigadas por instintos de sobrevivência, transpuseram-se para a dominação e o controle com o advento da agricultura. Contudo, as inovações tecnológicas decorrentes disso não extinguiram a necessidade da conexão entre elementos bióticos e abióticos condicionantes da homeostase dos ecossistemas, assim demandando, após curto período de afastamento - correspondente a menos de $5 \%$ da existência humana -, a retomada gradual de atitudes positivas vinculadas à natureza' ${ }^{1}$.

A defesa de tal concepção teve como pioneiro o biólogo norte-americano Edward Osborn Wilson, com a publicação da obra 'Biophilia', defendendo o mecanismo como inerente aos seres vivos, herdado após milhões de anos de coevolução junto às demais espécies² Quase dez anos depois disso, foi promulgada a Hipótese da Biofilia, que fundamentava a existência da necessidade humana de se relacionar com a natureza como meio de promoção de bem-estar biopsicossocial, colaborando, desta forma, para a constante valoração de parques, zoológicos, jardins, bosques, lagos e paisagens naturais, bem como a vinculação destes espaços com a sensação de paz e tranquilidade ${ }^{3}$.

A Declaração Universal sobre Bioética e Direitos Humanos ${ }^{4}$ considera que a identidade de um indivíduo compreende as dimensões biológica, psicológica, social, cultural e espiritual, incluindo suas relações com o ambiente. Logo, o fato de animais, plantas e paisagens estarem intrinsecamente inseridos na esfera relacional torna-os componentes essenciais na constituição da identidade dos seres humanos, do início ao fim de suas vidas 5 .

Os cuidados no fim da vida remediam inúmeras privações inerentes aos indivíduos que adoecem, e podem ser significativamente atingidos em suas esferas emocional e social6. Em 2002, a Organização Mundial da Saúde ${ }^{7}$ definiu nove princípios dos cuidados paliativos, sendo eles: I) promover o alívio da dor e de outros sintomas desagradáveis; II) considerar a morte como um processo natural; III) não acelerar nem adiar a morte; IV) integrar os aspectos psicológicos e espirituais no cuidado ao paciente; V) oferecer um sistema de suporte que possibilite ao paciente viver tão ativamente quanto possível até a sua morte; VI) oferecer sistema de suporte aos familiares durante a doença e o enfrentamento do luto; VII) abordagem multiprofissional em todas as etapas dos cuidados; VIII) melhorar a qualidade de vida e influenciar positivamente o curso da doença; e IX) iniciar o cuidado o mais precocemente possível.

Na prática de cuidados paliativos, a preservação da dignidade e da integridade do paciente constitui uma prioridade, incitando respeito à pessoa na sua plenitude, incluindo seus valores de vida. Para isto, tal prática intenciona mantê-lo conectado ao que tem sentido e valor em sua vida, mesmo diante da uma sequência de perdas ${ }^{8}$, considerando os valores ecológicos da pessoa como um direito fundamental e pretendendo manter a sua dignidade ${ }^{5}$.

A utilização da natureza como componente de manutenção metabólica é intrínseca a todos os seres vivos heterótrofos, cuja intermediação da evolução conduziu a seleção de determinados elementos para a restauração de processos biológicos, inserindo a relação da ação terapêutica de minerais, plantas e animais ${ }^{9}$. Nesse contexto, Fischer et al. ${ }^{9}$ refletiram sobre as questões éticas envolvidas no uso de animais zooterapêuticos como uma prática global enraizada culturalmente, porém, congregando uma pluralidade de agentes morais e vulneráveis. Os autores questionaram os limites e os cuidados envolvidos nas decisões de como e quando utilizar animais como recursos médicos. Fischer et al. ${ }^{10}$ analisaram, ainda, a zooterapia sob a perspectiva bioética, elencando, além dos 
inerentes benefícios, as vulnerabilidades que podem ser geradas caso a prática não seja intermediada por princípios bioéticos.

Os primeiros relatos da utilização de animais para práticas terapêuticas datam do início do século XVIII, quando pacientes de hospitais europeus portadores de transtornos mentais podiam passear e cuidar de jardins e animais domésticos ${ }^{11}$. Na década de 1960, Boris Levinson, psiquiatra infantil norte-americano, desenvolveu a psicoterapia facilitada por animais, visando tratar transtornos de comportamento ${ }^{12}$, e foi seguido por outros pesquisadores, que associaram benefícios aos animais de estimação no auxílio ao tratamento de pacientes crônicos ${ }^{13}$, culminando no registro como instrumento terapêutico ${ }^{\mathbf{1 4}}$. No Brasil, relatos da utilização de animais no contexto terapêutico psiquiátrico tiveram início na década de 1940, destacando-se o trabalho desenvolvido pela Dr $^{\text {a }}$ Nise da Silveira (Centro Psiquiátrico Pedro II, Rio de Janeiro). Houve também a oficialização da Terapia Assistida por Animais (TAA) em 1997, com a implementação do projeto filantrópico Pet Smile, pela psicóloga e veterinária Dra Hannelore Fuchs ${ }^{\mathbf{1 2}}$.

Diante da legitimidade da biofilia atestada por diferentes pesquisas avaliadas por Kellerte Wilson ${ }^{3}$ e Irvine e Warber ${ }^{15}$, o presente estudo questiona a exequibilidade da promoção da interação de pacientes em cuidados paliativos com elementos naturais no ambiente hospitalar. Para responder essa questão, testaram-se as hipóteses: a) a presença dos elementos naturais, em um momento de elevada vulnerabilidade, é reconhecido pelo meio científico como capaz de proporcionar benefícios na esfera biopsicossocial dos indivíduos; b) existem poucas evidências de promoção da interação do paciente em cuidados paliativos com os elementos naturais em decorrência da crença de sua ineficiência como medida terapêutica, diante da brevidade das intervenções; c) mesmo considerando que a interação com a natureza preveja o contato com elementos variados, como plantas, água, paisagens e frutas, acredita-se que haja prevalência da divulgação científica em Intervenções Assistidas por Animais (IAA), especialmente os cães.

Assim, objetivou-se analisar, por meio de revisão integrativa quanti e qualitativa, os efeitos do contato de elementos da natureza com pacientes sem possibilidade terapêutica curativa. Os resultados da pesquisa visam despertar novos olhares e condutas em relação aos métodos embasados na biofilia, com o intuito de suavizar e valorar o final da vida daqueles que se encontram sob cuidados paliativos. Foram analisados de acordo com princípios bioéticos de identificação e mitigação de vulnerabilidades em situações de conflito. Visou-se, assim, estabelecer um canal de comunicação entre agentes e pacientes morais, viabilizando soluções pautadas em valores comuns, com a mitigação do sofrimento e a valoração da vida em todas suas manifestações.

\section{Métodos}

O presente estudo constitui uma análise quanti e qualitativa de uma revisão bibliográfica integrativa ${ }^{16}$ no cenário científico nacional e internacional, tendo como pergunta norteadora a aplicabilidade de elementos naturais em pacientes sob cuidados paliativos, bem como as vulnerabilidades envolvidas com vias a promover uma reflexão à luz da bioética.

A contextualização acadêmica se deu por meio do levantamento de textos científicos veiculados na mídia digital, os quais detinham como eixo norteador a interação de pacientes sem possibilidade terapêutica curativa com elementos da natureza.

Os textos foram recuperados por meio do motor de busca Google Acadêmico, no período de agosto a novembro de 2017, sendo os resultados obtidos e analisados de acordo com duas perspectivas. Primeiramente, intencionando o mapeamento da relação entre biofilia e pacientes em cuidados paliativos, utilizando os descritores e seus respectivos registros no buscador: 'Cuidados paliativos e biofilia' ( $\mathrm{N}=2.304)$; 'Pacientes terminais 
e biofilia' ( $\mathrm{N}=242$ ); 'Fim de/da vida e biofilia' ( $\mathrm{N}=658)$; 'Doenças incuráveis e biofilia' ( $\mathrm{N}=497)$, em português e inglês, utilizando os mesmos no singular e no plural. A segunda etapa visou analisar a utilização de atividades que envolviam animais: 'Zooterapia e pacientes terminais' $(\mathrm{N}=715)$; 'Zooterapia e cuidados paliativos' ( $\mathrm{N}=813)$; 'Terapia com animais e pacientes terminais' ( $\mathrm{N}=209.500)$; 'Terapia com animais e cuidados paliativos' ( $\mathrm{N}=52.410)$; 'Interação animal e cuidados paliativos' ( $\mathrm{N}=7.070)$; 'Interação animal e pacientes terminais' $(\mathrm{N}=26)$; 'Animais e pacientes paliativos' ( $\mathrm{N}=14.699)$; e 'Animais e pacientes terminais' $(\mathrm{N}=3.790)$.

Para cada descritor, foram recuperados os primeiros cem resultados, os quais foram categorizados conforme: local, público, animais e elementos utilizados, e procedência da intervenção. Foram excluídos os resultados que não continham o conteúdo almejado, assim como os que se encontravam duplicados. A análise quantitativa testou a homogeneidade da amostra, comparando os parâmetros testados (elementos, benefícios e limitações) de acordo com as variáveis (nacional/internacional), partindo da hipótese nula da ocorrência nas mesmas proporções, aferidas por meio do teste do Qui-quadrado, considerando uma confiança de $95 \%$ e erro de $5 \%$.

A análise qualitativa se deu apenas no conteúdo específico, sendo cinco artigos correspondentes ao termo biofilia e sete à zooterapia, visando, por meio da técnica semântica do conteúdo de Bardin 17, elencar os pontos convergentes e divergentes apresentados nos estudos. A análise teve como linha norteadora os aspectos teóricos da Teoria da Biofilia ${ }^{2,3} \mathrm{e}$ os princípios dos cuidados paliativos ${ }^{7}$.

\section{Resultados e discussão}

\section{Biofilia e zooterapia no ambiente hospitalar em cuidados paliativos: análise quantitativa}

O conteúdo científico referente à caracterização da biofilia no contexto da assistência a pacientes em cuidados paliativos resultou em nove termos na contemplação total, uma vez que os resultados abarcavam menos de cem conteúdos, abrangendo $78 \%$ de análise dos retornos indicados pelo motor de busca. Foram recuperados 751 textos, sendo que, após a exclusão daqueles que não apresentaram conteúdo relativo, a interação entre biofilia e cuidados paliativos, bem como os duplicados, restaram 3,7\% ( $\mathrm{N}=28)$. Destes, 93\% traziam conteúdos atrelados ao ambiente hospitalar, prevalecendo o uso do jardim ecológico, de plantas e cães, destinados a um público geral e com predomínio do benefício emocional (figura 1).

A produção científica associada à zooterapia e pacientes sem possibilidade de cura resultou em sete termos contemplando toda a amostragem, culminando em uma abrangência equivalente a $45 \%$ de análise dos resultados. Foram recuperadas 1.177 referências, sendo que, após a exclusão, restaram $3,6 \%$ dos textos $(\mathrm{N}=43)$. A interação dos pacientes com animais voluntários de IAA foi identificada em $68,7 \%$ dos resultados, associada a alguma Organização Não Governamental (ONG), sendo que apenas $31,2 \%$ dos pacientes recebiam visitas de seus animais de estimação (figura 1). 
Figura 1. Síntese da revisão integrativa quantitativa sobre a inserção da biofilia e da zooterapia no ambiente hospitalar, em textos científicos nacionais (N) e internacionais (I)

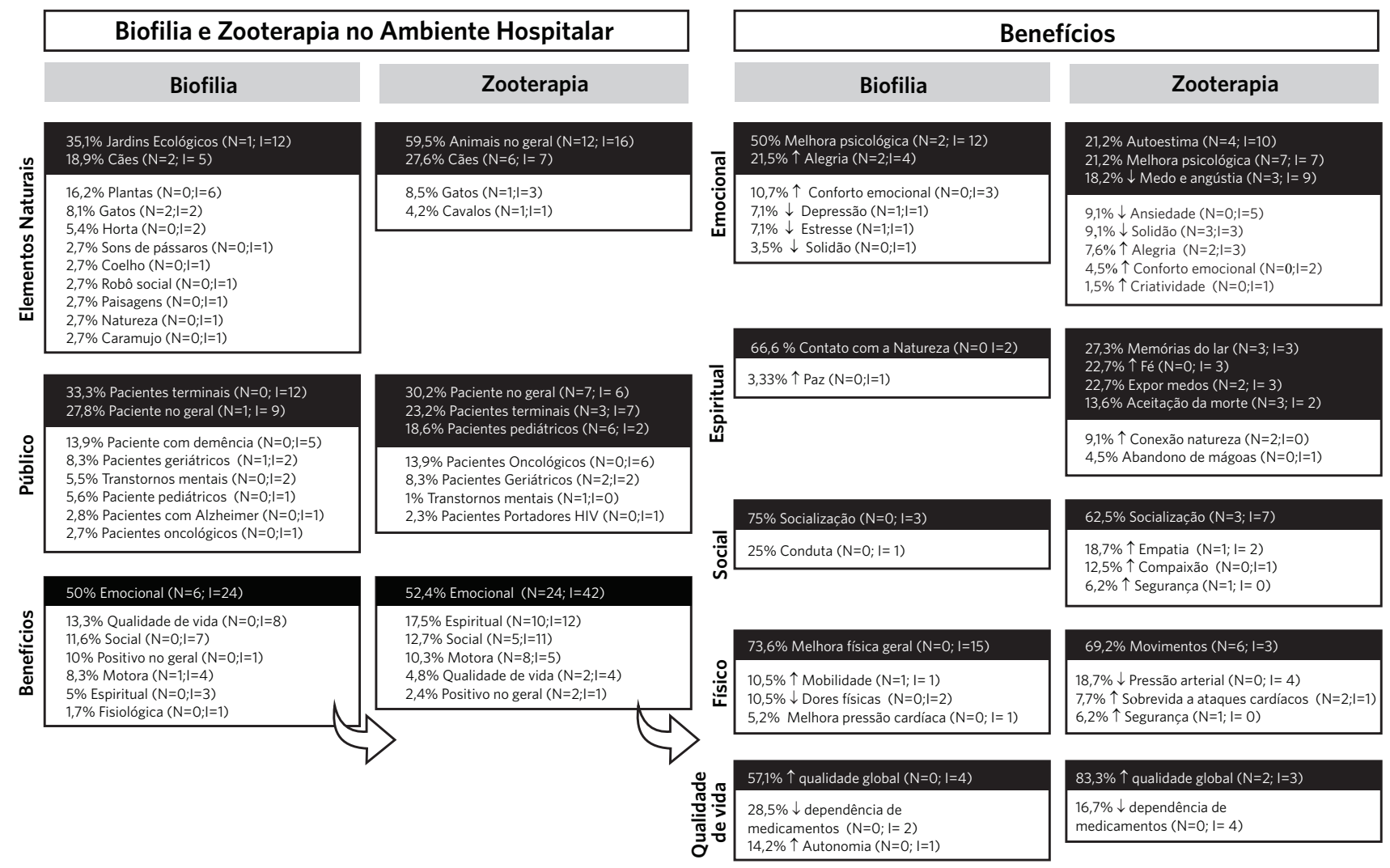

Fonte: Elaboração própria.

Nota: A homogeneidade dos resultados da categorização foi testada por meio do teste do Qui-quadrado, sendo os valores significativamente maiores ( $p<0,05$ ) destacados na caixa escura.

Os dados obtidos na análise quantitativa contrariaram a expectativa da hipótese de prevalência de cães em atividades assistidas, em pacientes em fase de terminalidade, destacando-se o uso de jardins ecológicos. Contudo, ressalva-se que ainda associado a práticas desenvolvidas no cenário internacional. O presente estudo evidenciou a hipótese inicial da importância biopsicossocial estabelecida através do contato entre pacientes e elementos naturais, revelando aspectos positivos em praticamente todas as esferas analisadas, possuindo maior evidência quando relacionada ao emocional. Isto corrobora a pesquisa de Muschel ${ }^{\mathbf{1 8}}$, envolvendo pacientes em fase final, que atestou a diminuição de sentimentos de desespero, solidão e estresse causados pela proximidade da morte, em decorrência da presença e estimulação táctil de um animal, alcançando a expectativa de cuidado necessariamente não atrelado à cura ${ }^{19}$.

\section{Biofilia e zooterapia como práticas terapêuticas em cuidados paliativos: análise qualitativa}

A análise qualitativa associada à inserção da biofilia como prática terapêutica em cuidados paliativos atestou $57,1 \%$, referindo-se apenas às citações, sendo que metade daqueles que tratavam dos cuidados paliativos como uma das abordagens principais correspondeu a 
relatos de casos ou experimentação prática, e a outra se limitou a reflexões teóricas. A maioria do conteúdo recuperado, associado à zooterapia, consistia de pesquisas teóricas $(55,8 \%)$ e abordava a temática de maneira superficial. Dos estudos específicos, 13,9\% tratavam de reflexões teóricas. Entre as instituições que permitiram a visitação de animais, predominou a intermediação por voluntariado (81,3\%), enquanto $18,6 \%$ dos pacientes tiveram a oportunidade de interagir com seus animais de estimação (figura 2).

Os textos associados diretamente à biofilia no contexto de cuidados paliativos trouxerem duas abordagens: interesse clínico (64,3\%) e interesse da arquitetura (35,7\%). Ambos trataram a biofilia como aspecto fundamental na qualidade de vida de pacientes, familiares e equipe terapêutica (figura 2).

Os conteúdos de abordagem médico-clínica focaram em aspectos terapêuticos da biofilia, promovida pela interação com animais, plantas e jardins, sendo estes dois últimos os mais frequentes. Já os benefícios associados às IAA corroboram as evidências associadas à melhora da autoestima, dos sintomas depressivos e da qualidade de vida ${ }^{10}$. Outros estudos trouxeram novidades, tais como a horticultura terapêutica, reportada por Wallis e Lenon ${ }^{\mathbf{2 0}}$ como uma intervenção não medicamentada, na qual os pacientes em cuidados paliativos podem apoiar uns aos outros.

Já a abordagem inerente à arquitetura demonstrou enfoque nos benefícios alcançados pela biofilia, principalmente a partir dos aspectos contemplativos, e na promoção de ambientes salutogênicos para a melhoria da saúde e do bem-estar. Erickson ${ }^{21}$ relacionou a teoria dos Jardins de Cura, de Roger Ulrich ${ }^{22}$, como um potencial benefício para a saúde dos pacientes em geral. Segundo a teoria, a imersão nos jardins de cura promove suporte social, privacidade, opções e locais para momentos reservados, além de estimular movimentos físicos, fundamentais para a reabilitação, e distrações por meio de plantas, flores, água, vida selvagem e sons da natureza. Dalton e Harrisson ${ }^{\mathbf{2 4}}$ salientaram, também, o 'princípio da congruência' e a 'teoria do modelo salutogênico' no design, justificando que a falta de um espaço de convívio coerente (sem congruência) pode levar as pessoas a adoecerem. Os autores apresentaram a proposta de um 'quarto personalizável', que otimizasse a congruência entre os usuários e um meio saudável, contemplando aspectos biofílicos. 
Figura 2. Síntese da análise qualitativa dos textos recuperados, com abordagem da inserção da biofilia e da zooterapia nos cuidados paliativos, e quantificação dos textos que atendiam aos princípios dos cuidados paliativos

Biofilia e Zooterapia como prática humanizadora dos cuidados paliativos

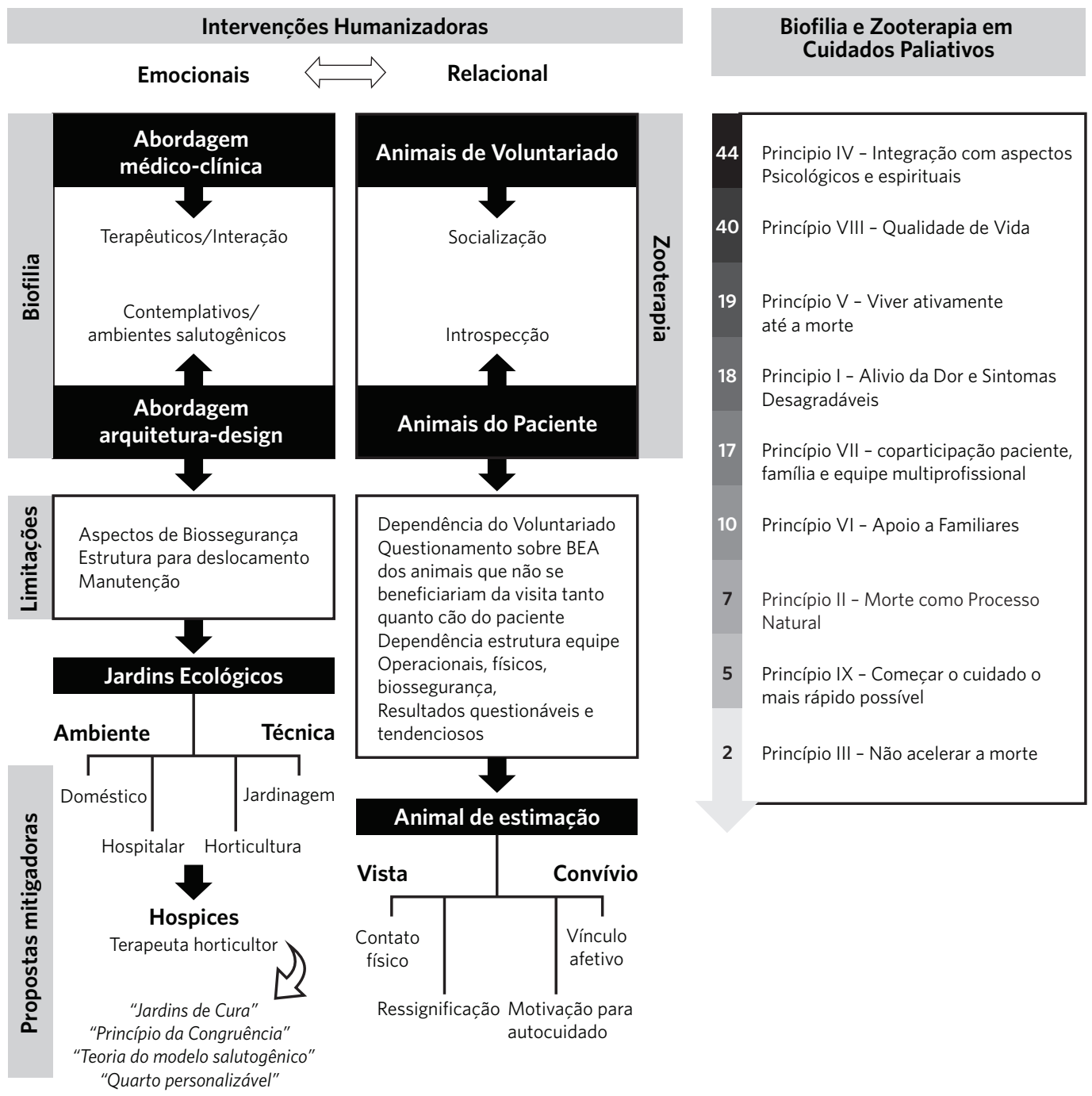

Fonte: Elaboração própria a partir das referências $\mathbf{1 8 , 2 0 , 2 4 - 3 3}$.

Em todos os textos analisados, foi possível identificar benefícios da aproximação de pacientes com os elementos naturais, corroborando a hipótese testada no presente estudo e associada à Hipótese da Biofilia ${ }^{3}$, pronunciando aqueles de cunho emocional, seguido dos aspectos sociais e atrelado à qualidade de vida. A interação com animais, especialmente cães, esteve mais relacionada aos benefícios sociais, da esfera relacional, como a diminuição da solidão, auxílio na comunicação e na conduta social ${ }^{34}$. Por sua vez, a interação com jardins ecológicos se relacionou aos benefícios emocionais, como a melhora do humor e a diminuição do estresse e da depressão (figura 2). Queiroz $^{35}$ relatou que a maioria dos 
pacientes participantes do seu estudo convivia com animais de estimação. Enquanto Wallis e Lenon ${ }^{20}$ retrataram que pacientes que possuíam jardim em suas residências, mas passaram a viver em leitos de hospitais, relataram que adorariam poder sair ao ar livre novamente. Segundo os autores, um

paciente moribundo em cuidados paliativos, que havia, anteriormente, plantado uma semente de agrião, sorriu ao sentir o perfume da planta que foi segurada sob suas narinas 20(25).

Contudo, mesmo diante de dados que atestam a efetividade da prática, elencam-se limitantes da inserção de jardins em hospitais, que podem ser de ordem logística, tais como a necessidade de equipes de manutenção, terapêuticas, de atendimento ao deslocamento e, ainda, o aumento dos riscos que um jardim pode proporcionar, tais como a aproximação de insetos e animais, como formigas, mosquitos e pombos, os quais potencialmente podem aumentar os riscos de biossegurança nos hospitais ${ }^{36}$.

As IAA são práticas que vêm sendo mundialmente implantadas como medidas de humanização em ambientes hospitalares, com predominância no setor público, inclusive, nos cuidados paliativos. Engelman ${ }^{\mathbf{3 7}}$ utilizou animais terapeutas em um ambulatório de cuidados paliativos durante um ano, observando que os pacientes ficavam mais felizes e estimulados, reduzindo emoções negativas mediadas pela verbalização de boas recordações. De forma geral, sentiam-se relaxados e mencionavam que os animais traziam-lhes conforto para o enfrentamento das doenças. A melhoria da qualidade de vida do paciente em cuidados paliativos foi atestada por Turnbach ${ }^{33}$, ressaltando que os benefícios transpassavam os pacientes, promovendo uma experiência positiva antiestresse para a equipe terapêutica e os membros da família. Corroborando esses dados, Muschel18 desenvolveu um estudo com pacientes em tratamento oncológico, para os quais a presença dos animais constituiu recursos redutores de estresse. Apesar dos resultados das IAA ainda serem questionados quanto a análises tendenciosas, recebendo críticas severas aos métodos de análise, além da constante alegação de vazio teórico ${ }^{38}$, percebe-se que, de modo geral, despertam sentimentos positivos em pacientes, inclusive, os que se encontram sem possibilidade terapêutica curativa ${ }^{\mathbf{2 8}}$.

Nos artigos analisados, foi recorrente a citação do estudo 'Pet therapy with terminal cancer patients', de autoria de Irene J. Muschel, uma ativista norte-americana que busca resgatar conceitos de cuidados com os animais. No artigo, Muschel18 (1984) entrevistou pacientes com doenças incuráveis avançadas, semanalmente expostos a cães por uma hora e meia, durante dez semanas. A presença dos animais supria necessidades psicológicas e sociais dos pacientes, até então impossíveis de serem atendidas. Um destaque foi dado à diminuição de sentimentos associados ao medo, ao desespero, à solidão e ao isolamento. Além disso, propiciou um auxílio na aceitação de sentimentos relativos à inevitabilidade da morte. Os pacientes que participaram da pesquisa por meio de questionário indicaram que a presença dos animais diminuía a sensação de desamparo, sendo assim, permanentemente clamavam pela presença deles. A correlação entre a diminuição da solidão e a tutela de um animal foi igualmente reconhecida pela autora como benéfica para determinar a qualidade de vida dos pacientes.

No contexto da zooterapia, foram identificados dois encaminhamentos: os animais de voluntários e os animais dos pacientes. Enquanto no primeiro desponta claramente a promoção de socialização do paciente ${ }^{39}$, o caso da interação com o próprio animal de estimação promove introspecção e diálogo interno. No contexto terapêutico, para pacientes no geral $^{39} \mathrm{e}$, inclusive, para a população idosa não hospitalizada ${ }^{\mathbf{4 0}}$, crianças $^{\mathbf{2 8}} \mathrm{e}$ encarcera$\operatorname{dos}^{41}$, ambas as abordagens são atestadas como benéficas para a saúde global, sendo destacada a possibilidade de conforto psicológico 
promovido pelo contato físico destituído de julgamentos e limitantes sociais ${ }^{10,34}$. No entanto, no contexto dos cuidados paliativos, a oportunidade de elaborar o momento de finitude tem uma relação significativa, quando o processo de contato se faz com o próprio animal ${ }^{\mathbf{4 2}}$.

Embora a zooterapia seja reconhecida como uma eficiente prática de interação entre humanos e animais, as instituições elencam uma série de limitantes operacionais, principalmente, quanto ao aumento de riscos envolvidos com a biossegurança ${ }^{10}$, mesmo diante da insuficiência de estudos sobre a exposição de pacientes e animais a possíveis zoonoses e patógenos. Para Murthy, Pandrangi e Welber ${ }^{\mathbf{4 3}}$, os resultados obtidos até o momento são baseados em relatos de caso e investigações de surtos e, portanto, não podem mensurar de forma eficaz a possibilidade de risco aos envolvidos. Acrescente-se a isso, a argumentação de pesquisadores da área do Bem-Estar Animal (BEA), que clamam por condutas éticas e pela normatização do uso dos animais, ainda que sejam elencadas algumas vulnerabilidades, como a exaustão destes, em razão de muitas horas de trabalho; aumento de ansiedade, por conta da exposição a odores e locais diferentes; incompatibilidade de características físicas e psicológicas dos animais à prática; inexistência de protocolos consolidados e compatíveis com a realidade, que envolvam treinamento certificado e padronizado; e, principalmente, a dificuldade para lidar com essas limitações em função da carga emotiva atrelada à justificativa do uso do animal envolvida no trabalho solidário ${ }^{\mathbf{1 0}, 43,44}$. Embora em muitos países já existam protocolos para zooterapia, no Brasil, a regulamentação fica a cargo de cada instituição, sendo predominantemente conduzida pelo terceiro setor ${ }^{\mathbf{1 0}, 44}$.

\section{Biofilia e zooterapia: congruência com os princípios dos cuidados paliativos}

Em todos os textos resultantes da revisão integrativa, foi possível identificar um ou mais princípios dos cuidados paliativos (figura 2).

Os princípios I, IV e VIII, que intentam aliviar a dor e outros sintomas desagradáveis, incluindo-se os relacionados a fatores psicossociais e espirituais, visando à melhoria da qualidade de vida, foram identificados em 18,40 e 44 artigos, respectivamente. Estes resultados atestam que medidas não farmacológicas auxiliam esses propósitos, pois tendem a transformar o clima hostil, relacionado à enfermidade e à proximidade da morte, em um ambiente descontraído e agradável ${ }^{28}$. Partindo da concepção da dor como um processo subjetivo e plausível de interferências de características individuais e do estado emocional e espiritual10,45,46, é concebível que a interação com outro ser vivo interfira positivamente, como atestado por Varas ${ }^{47}$, que registrou uma percepção da dor até quatro vezes menor nos pacientes envolvidos.

A presença do animal e de outros elementos naturais promove a mudança do foco egocêntrico e eleva a disposição para se comunicar ${ }^{19,32,39}$, amenizando as sensações de dor, medo, angústia e depressão ${ }^{24}$ dos pacientes, melhorando-lhes o humor ${ }^{48}$ e a autoestima ${ }^{35}$, e diminuindo-lhes comportamentos agressivos ${ }^{15}$. Alguns pacientes se sentiram conectados aos animais ${ }^{\mathbf{2 8}}$, pois, sob a perspectiva emocional, a presença destes promove conforto, visto que não é autoritária e se oferece destituída de julgamentos, em momentos nos quais, muitas vezes, os familiares evitam falar sobre a morte eminente, como forma de se protegerem do sofrimento. A isto, acrescenta-se ao paciente o sentimento de responsabilidade e zelo pelo outro, e de ser amado e importante para o outro ${ }^{49}$.

A melhoria da qualidade de vida do paciente em cuidados paliativos demanda a contemplação do ser humano a partir de uma abordagem holística $^{8}$ e ecológica ${ }^{5}$, constituindo-se a interação com o mundo natural como uma parte vital do bem-estar biopsicossocial e espiritual do indivíduo ${ }^{15}$.

Os princípios II, III, IV e IX aspiram à promoção da afirmação da vida, e da concepção da morte como um processo natural, valorizando 
cada momento que ainda pode ser vivido o mais ativamente possível, buscando meios de não acelerar nem adiar a morte ${ }^{\mathbf{4 7}}$, tendo como desafio a superação da linha tênue entre o fazer e o não fazer ${ }^{44}$. Foram identificados em 8, 2,15 e 5 artigos, respectivamente. A utilização de elementos naturais neste processo promove o resgate de sentimentos positivos, muitas vezes difíceis de serem estabelecidos com/ entre familiares ou amigos, pois a necessidade de elaboração dos últimos momentos de vida envolve tensão, medo e insegurança diante da morte iminente, podendo comprometer a qualidade desse período ${ }^{28}$.

De igual modo, a contemplação de paisagens e a interação com animais e plantas durante as mudanças de estação, além da própria identificação da morte na natureza, inserida nos ciclos naturais curtos ou longos, e a compreensão do fim e do recomeço, podem ser os fatores determinantes para a aceitação da morte como um processo natural, tal como evidenciado por Wallis e Lenon ${ }^{20}$ na prática da horticultura terapêutica. Essa prática foi relatada por Leckie e Pilgrem ${ }^{27}$ e Wallis e Lenon ${ }^{20}$ como benéfica para pacientes em cuidados paliativos, ao promover a afirmação da vida e a preparação para a morte, sem que necessariamente a iminência da mesma esteja permanentemente presente. Os autores relataram casos nos quais o cultivo dos vegetais era realizado ao lado da cama dos pacientes, proporcionando-lhes, até o último momento, a oportunidade de colaborar com a continuidade da vida. O encantamento diante de outro ser vivo pode incitar movimentos, deter a atenção e comunicar isso a terceiros, tal como o aumento do nível de alerta cognitivo em pacientes paliativos pediátricos registrado por Varas ${ }^{47}$. Para Lima e Sousa ${ }^{39}$, os benefícios sociais estão na destituição de conceituações, fazendo com que os pacientes não se incomodem com suas próprias limitações físicas e mentais, permitindo-lhes uma aproximação com os outros e desenvolvendo neles a confiança para realizar atividades que demandem coordenação motora. Isso transpassa uma intenção de simplesmente prolongar a vida, mas visa proporcionar qualidade de vida e bem-estar, preservando a identidade por meio da inserção da dimensão ecológica do sujeito no aspecto da ressignificação da vida e da representação do que é estar vivo ${ }^{8}$.

A promoção de terapias alternativas, como o cultivo de plantas e a interação com animais, ou simplesmente o despertar da fascinação por árvores, rochas, plantas e demais componentes da vida selvagem pode elevar o espírito a outro patamar de contemplação, provendo um sentido de estar longe e transpassando o plano da doença ${ }^{3}$. Segundo o manual da Academia Nacional de Cuidados Paliativos ${ }^{50}$, a abordagem precoce permite a prevenção dos sintomas e de complicações inerentes à doença de base $\mathbf{4 4}^{4}$, as quais encontram suporte nas intervenções com terapias alternativas como horticultura, jardinagem ou IAA, principalmente, de ordem emocional $\mathbf{2 0 , 2 1 , 2 2}^{2}$.

Os princípios VI e VII instituem auxílio aos familiares durante a doença e no enfrentamento do luto, e a coparticipação de pacientes, familiares e equipe profissional no processo de cuidados paliativos ${ }^{\mathbf{1 8}}$, sendo identificados em 10 e 17 artigos, respectivamente. Lima e Sousa ${ }^{39}$ apontaram que os animais, além de proporcionarem condições relacionadas a apoio, companhia e conforto aos pacientes, são hábeis em impactar positivamente os familiares e as equipes profissionais, que, igualmente, se encontram em períodos de vulnerabilidade emocional. Esses resultados foram atestados por Varas ${ }^{\mathbf{4 7}(\mathbf{2 0 1 0})}$, relacionados a tutores de crianças sob cuidados paliativos, e por Turnbach ${ }^{33}$, na qualidade de vida dos familiares e cuidadores durante o período de aceitação da doença, visto que houve melhoras no apoio e enfrentamento da situação. Erickson ${ }^{21}$ relatou que os jardins de instituições de cuidados paliativos proporcionam ambientes mais acolhedores e reconfortantes para familiares e pacientes interagirem. As áreas reservadas oferecem privacidade para meditação e reflexão na contemplação da natureza, por vezes, fundamentais no enfrentamento do luto. Ressalta-se que o animal do paciente e/ 
ou uma planta por ele cultivada representam, para o familiar, um elo que o mantém vivo em outros seres, que carregam a sua existência, podendo esta ser resgatada, o que traz conforto e, incontestavelmente, legitima a prática de humanização em cuidados paliativos. Tudo isto traz à tona a necessidade de abordagens interdisciplinares que acessem não somente as necessidades clínicas dos pacientes, mas também suas necessidades psicossociais e espirituais, assim como as de seus familiares.

A presença do elemento natural facilita ao enfermo externar seus medos, sentir-se mais confiante para falar de sua situação e, desta forma, abordar assuntos com a equipe médica e familiares, talvez nunca antes mencionados $^{51}$. Muschel18 identificou que a presença de animais deixou profissionais e familiares mais relaxados, com menos desconforto diante das situações, pois permitiu o compartilhamento de lembranças positivas. Por isso, Kawakami e Nakano ${ }^{51}$ reforçaram a necessidade de que a IAA e demais intervenções terapêuticas biofílicas sejam partilhadas e construídas conjuntamente à equipe terapêutica, a fim de que esta se insira no contexto, seja hábil em avaliar as consequências do tratamento e colabore com a eficiência da técnica. Ressalva-se que, no ambiente hospitalar, o paciente dispende mais tempo com a equipe médica do que com os familiares. Automaticamente, o sucesso das intervenções nos cuidados paliativos deve prever um acolhimento dos profissionais. Segundo Matsumoto ${ }^{44}$, a família - tanto a biológica como a adquirida, congregando amigos, parceiros e os próprios animais de estimação - pode e deve ser parceira e colaboradora no cuidado, pois o conforto e o apoio emocional proporcionado podem ser tão significativos quanto o suporte médico. Logo, a visitação do animal de estimação do paciente pode encontrar suporte argumentativo nos princípios VI e VII, uma vez que proporcionam apoio à família quando legitimam manter o paciente ligado a tudo que dá sentido à sua vida, corroborando o argumento de Bertachini e Pessini ${ }^{8}$, de que preservar a identidade do paciente é uma prioridade em cuidados paliativos.

\section{Mitigando as vulnerabilidades: pro- moção da biofilia em hospice como um meio ético de cuidados paliativos}

Os principais fatores limitantes para a prática da biofilia em ambientes hospitalares referiram-se a questões de biossegurança acrescidas de logística para sua manutenção (figura 2). Diante da legitimidade deste argumento e de resultados positivos relatados na literatura, propõe-se a promoção de terapias biofílicas em hospices como meio ético de promoção de cuidados paliativos.

A morte, ao longo da história da humanidade, foi associada a sentimentos ruins e desconfortáveis, resultando em pacientes solitários, com autonomia enfraquecida e incapazes de vislumbrar sentidos para suas existências ${ }^{52}$. Contudo, esta é uma preocupação que subsiste desde a Idade Média, cujos monastérios ofereciam acolhimento a famintos, pobres, órfãos, leprosos e moribundos $^{50}$. O movimento denominado Filosofia Hospice, originado na Inglaterra, no século $\mathrm{XX}^{\mathbf{4 2 , 5 3}}$, propunha minimizar essa vulnerabilidade por meio de uma assistência humanizada, cujas práticas transpassavam diagnósticos, tratamentos e prognósticos, vislumbrando o cuidado holístico, envolvendo as esferas física, emocional, social, espiritual e, mais recentemente, financeira, interpessoal, familiar e da equipe profissional.

O modelo de cuidados paliativos começou a ser debatido e implantado no Brasil somente no início da década de 1980, contudo, os métodos eram baseados em cuidados à saúde essencialmente curativos, e apenas se davam no ambiente hospitalar ${ }^{\mathbf{4 2}}$. Rodrigues e Zago $\mathbf{4 2}^{\mathbf{2}}$ relataram o movimento, adaptando a Filosofia Hospice à realidade brasileira, superando a concepção de que não havia nada a ser feito pelos pacientes em fase terminal, em função da convicção de que muito pode ser feito, partindo do princípio de que, enquanto há vida, existe a possibilidade do cuidado ${ }^{19}$. No contexto dos cuidados paliativos, sentimentos de empatia e compaixão são fundamentais para as pessoas que se encontram em estados de terminalidade de vida $\mathbf{4 2}^{\mathbf{2}}$ e vulneráveis diante 
de alterações físicas, emocionais e existenciais ${ }^{54}$. Assim, as instituições de hospices são designadas para serem locais de descanso, invocando o conforto e a sensação de estar em casa, alcançada, segundo Erickson ${ }^{21}$, com a existência de lindos jardins, por exemplo. Segundo a autora, pacientes bem acomodados em camas móveis aproveitam a natureza sem perder o conforto ou a assistência de seus leitos, favorecidos pelo fato de os sentidos da audição e do olfato, amplamente estimulados pelo jardim, poderem ser os últimos a desaparecer. Igualmente, se pressupõe a existência de locais solitários para a meditação e a contemplação da natureza, bem como locais para que pequenos grupos de familiares possam se reunir e conversar ${ }^{21}$. Logo, alpendres, varandas, sacadas e átrios promovem uma sensação aconchegante, além de facilitar a transição entre estar dentro da instalação e ao ar livre ${ }^{21}$. Segundo Sadler ${ }^{55}$, quando disponível, o jardim é um refúgio seguro e protegido, que normalmente circunda a estrutura predial, oferecendo uma maravilhosa vista para os pacientes. Tais jardins são experiências mentalmente restauradoras para os pacientes, familiares e profissionais, que passam muito tempo nessas instituições. $\mathrm{O}$ arquiteto Ulrich ${ }^{56}$ ressaltou que existem evidências consideráveis de que os efeitos restaurativos da contemplação da natureza se manifestam dentro de três a cinco minutos, decorrentes de uma sequência de alterações fisiológicas. Considerando o aspecto psicológico/emocional, as observações da natureza ou de jardins elevam os níveis de sentimentos positivos e reduzem emoções negativamente tonificadas, como medo, raiva e tristeza. Certos cenários da natureza efetivamente sustentam o interesse e a atenção, e, consequentemente, servem como agradáveis distrações, que podem diminuir pensamentos estressantes. Quanto às manifestações fisiológicas de recuperação do estresse, investigações laboratoriais e clínicas atestaram que a contemplação da natureza também pode produzir restauração significativa em menos de cinco minutos, como, por exemplo, melhora na pressão arterial, na atividade do coração, na tensão muscular e nas atividades cerebrais ${ }^{56}$.

Inúmeras instituições já despontam no cenário internacional por implementarem programas de jardinagem e horticultura, nos quais pacientes monitorados por equipes multidisciplinares - compostas por terapeutas horticultores, psicólogos, enfermeiros e fisioterapeutas - plantam mudas, que ganharão seus nomes e serão transplantadas para o jardim $^{57}$. O medo da finitude é a maior barreira enfrentada no processo de elaboração da aceitação da morte, e transpor a existência para uma planta pode ser considerada uma prática humanizadora, uma vez que traz a sensação de continuidade. Os mesmos mecanismos são percebidos no familiar, que vê, na árvore plantada pelo seu ente querido, o fluxo da vida. Esta prática, evitada em hospitais que primam pela segurança biológica, encontra no hospice um local ideal para ser desenvolvida. A prática de jardinagem como coadjuvante em processos terapêuticos, que visam a uma maior qualidade de vida, já é constatada ${ }^{55}$. Erickson ${ }^{21}$ transpôs a amplitude dessa prática milenar, ao sugerir a presença de jardins terapêuticos em hospitais de cuidados paliativos, pois alguns pacientes poderiam aproveitar o tempo ao ar livre, mesmo estando acamados.

A horticultura terapêutica, embora esteja documentada na literatura, se constitui de uma proposta relativamente nova em hospices, demandando adaptações às necessidades e limitações dos pacientes, sendo realizada, muitas vezes, suspensa em mesas e em vasos ${ }^{21}$, ou até mesmo na cabeceira das camas ${ }^{20}$. Segundo Leckie e Pilgrem ${ }^{27}$, e Kumasaka et al.48, esta prática se constitui como um meio de aumentar a qualidade de vida, por aliviar o sofrimento e os sintomas de demência; reduzir a percepção da dor; melhorar o humor, as habilidades cognitivas, motoras e da fala; e promover uma reabilitação, fazendo com que os pacientes vivam plenamente até os últimos dias de suas vidas ${ }^{27}$. 


\section{Mitigando as vulnerabilidades: a visita do animal de estimação do paciente como uma prática ética nos cuidados paliativos}

Com relação à zooterapia, a questão emergente é a existência de diferenças da visita de animais de voluntários e de animais de estimação. Considerando que $44 \%$ das famílias brasileiras possuem animais de estimação ${ }^{58}$, atesta-se o espaço que lhes é destinado junto às famílias. Logo, o valor desse vínculo afetivo deve ser considerado no cuidado de pacientes em cuidados paliativos. Entre os inúmeros benefícios, desponta a relação baseada na doação e destituída de julgamentos, preconceitos e estigmas, acrescida pelo fato de o animal prover o contato físico tão necessário para o bem-estar biopsicossocial12 e espiritual do paciente. Ilustrando esta situação, Rocha ${ }^{59}$ descreveu o desejo de um jovem paciente, em estado terminal de vida, de receber a visita de seu animal de estimação, alegando que, nas horas em que se encontrava sozinho, o animal era seu único e inseparável companheiro, sentindo-se confortado e cuidado por ele. Assim, a partir da visitação de animais de estimação a pacientes internados em um hospital oncológico, o autor evidenciou que, além da redução de sintomas negativos, aqueles se tornavam referência, fonte de intimidade e ressignificação do adoecer e da vida para os pacientes. Relatos colhidos ainda por Rocha ${ }^{59}$ pontuaram que a oportunidade de interagir com seus animais proporcionaram efeitos terapêuticos reparadores em todos os pacientes, uma vez que eles passaram a se mostrar mais dispostos a participar de decisões referentes a seus tratamentos. Tais decisões, mesmo não sendo curativas, tinham o intuito de melhorar o condicionamento dos pacientes, a fim de receberem seus animais de estimação com mais disposição $0^{60}$. Segundo $\operatorname{Costa}^{40}$, para pacientes que não partilham de relações familiares com outras pessoas, os animais são constantes fontes de conforto e intimidade, representando chances de cuidarem de outros seres. Outra questão ética a ser considerada, referente a muitos pacientes idosos, é o fato de que não têm ninguém para cuidar de seus animais, desencadeando momentos de angústia que interferem no seu bem-estar. A utilização do animal do próprio paciente configura, ainda, uma questão de BEA, uma vez que o reencontro proporciona benefícios também ao animal, que sente falta de seu tutor.

\section{Conclusões}

Os resultados do presente estudo trazem um panorama da inserção de elementos naturais nas práticas de cuidados paliativos, atestando a hipótese de que, embora os relatos das intervenções ainda sejam incipientes, já é possível vislumbrar a sua legitimidade. Entretanto, são necessárias reflexão ética e avaliação científica nos cuidados e prioridades, no fim da vida, sob a perspectiva bioética, a fim de minimizar as vulnerabilidades inerentes a este período de defrontação com alguma doença incurável. A prática promove a sinergia dos princípios dos cuidados paliativos, balizados pelo suporte aos aspectos psicológicos e espirituais, e a melhora da qualidade de vida dos pacientes, enaltecendo, principalmente, os benefícios emocionais da biofilia e da zooterapia.

A partir dos dados do presente estudo, foi possível atestar a hipótese de que os benefícios decorrentes da intrínseca e natural inter-relação entre os seres humanos e os elementos naturais são efetivos em momentos de elevada vulnerabilidade, se mostrando hábeis na promoção de benefícios nas esferas biopsicossocial e espiritual. Isto porque, correspondendo aos princípios dos cuidados paliativos, intencionam o oferecimento de um sistema de suporte para que os pacientes vivam o mais ativamente possível até sua morte. Acrescido da integração dos aspectos psicológicos, sociais e espirituais ao clínico, este suporte transpõe a crença inicial da insuficiência da prática frente à brevidade das intervenções. 
Os resultados atestam a hipótese de eficiência de métodos usuais, como a zooterapia com cães, passando pela inserção de métodos incomuns no cenário nacional, como a promoção do convívio com elementos naturais, como plantas, água, paisagens e frutas. A reflexão do conteúdo científico analisado permitiu sugerir a inserção da horticultura terapêutica em hospices e criar programas para visitas dos animais de estimação. Os resultados da pesquisa permitem fomentar novos olhares e condutas em relação aos métodos embasados na biofilia, com o intuito de suavizar e valorar o final da vida daqueles que se encontram sob cuidado.

A análise da questão sob a perspectiva da bioética permite a identificação de vulnerabilidades inerentes a esse momento de finitude - que impactam igualmente em familiares e equipe médica -, nos quais a inserção de elementos biofílicos se constitui como um meio de mitigação dessas condições. Uma vez que promove um canal de comunicação entre os atores envolvidos nesse cenário e a confluência de valores comuns, visa à valoração da vida e do não sofrimento de todos os seres vivos. Assim, na manutenção da identidade e integridade dos pacientes em cuidados paliativos, a biofilia é um importante aspecto a ser considerado.

Para algumas pessoas, cuidar de um jardim, interagir com animais ou, simplesmente, contemplar a natureza, pode envolver valores que não encontram equivalência a serem respeitados como práticas de dignidade. No entanto, ao se considerar a dimensão ecológica de um indivíduo e os valores ambientais que o compõem, compreende-se a esta dignidade a ser respeitada e preservada. Tal dimensão é expressa pela biofilia como uma necessidade inata de afiliar-se com outras formas de vida, e não pode ser desconsiderada na prática dos cuidados paliativos. Ressalva-se que a indicação e o estímulo ao convívio salutar com elementos naturais podem compor as agendas educacionais e de saúde bem antes da doença surgir, prevenindo sua manifestação, colocando em prática a Hipótese da Biofilia.

\section{Colaboradores}

Zanatta AA (0000-0001-8390-1573)* contribuiu para a concepção, o planejamento, a análise e a interpretação dos dados. SantosJunior RJ (0000-0003-3724-8467)* contribuiu para a concepção, o planejamento, a análise e a interpretação dos dados. Perini CC (00000002-9340-8704)* contribuiu para a concepção e o planejamento do estudo. Fischer ML (0000-0002-1885-0535)* contribuiu para a concepção, o planejamento, a análise e a interpretação dos dados. 


\section{Referências}

1. Salisbury J. The beast within: Animals in the Middle Ages. London: Routledge; 2012.

2. Wilson EO. Biophilia. Cambridge: Harvard University Press; 1984.

3. Kellert SR, Wilson EO. The biophilia hypothesis. Washington: Island Press; 1995.

4. Organização das Nações Unidas para a Educação, Ciência e Cultura. Declaração Universal sobre Bioética e Direitos Humanos. Lisboa: Unesco; 2006 [acesso em 2018 ago 10]. Disponível em: http://unesdoc. unesco.org/images/0014/001461/146180por.pdf.

5. Sousa PHM. A dimensão ecológica da dignidade humana. [dissertação]. Curitiba: Universidade Federal do Paraná; 2011. 161 p.

6. Singer PA, Martin DK, Kelner M. Quality end-of-life care: patients' perspectives. Jama. 1999; 281:163-168.

7. Krug EG, Dahlberg LL, Mercy JA, et al. Relatório mundial sobre violência e saúde. [internet]. Genebra: Organização Mundial da Saúde; 2002 [acesso em 2018 ago 2]. Disponível em: https://www.cevs. rs.gov.br/upload/arquivos/201706/14142032-relatorio-mundial-sobre-violencia-e-saude.pdf.

8. Bertachini L, Pessini L. A importância da dimensão espiritual na prática dos cuidados paliativos. Rev. Bioetikos. 2010; 4(3):15-23.

9. Fischer ML, Palodeto MFT, Santos EC. O uso de animais como zooterápicos: uma questão bioética. Hist. ciênc. saúde-Manguinhos. 2018; 25:217-243.

10. Fischer ML, Amorim AZ, Rezende EA. Um olhar da bioética para a zooterapia. Rev. latinoam. bioét. 2016; 16:174-197.

11. Kruger KA, Serpell JA. Animal-assisted interventions in mental health: Definitions and theoretical foundations. In: Fine AH, editor. Handbook on animal-assisted therapy: Theoretical foundations and gui- delines for practice. London: Academic Press; 2010. p. 133-148.

12. Chelini MOM, Otta E. Terapia Assistida por Animais. Barueri, SP: Manole; 2016.

13. Nightingale F. Notas sobre a enfermagem: o que é e o que não é. Rev. bras. enferm. 1989; 4:109-118.

14. Hooker SD, Freeman LH, Stewart P. Pet therapy research: a historical review. Holist. nurs. pract. 2002; 17:17-23.

15. Irvine KN, Warber SL. Greening healthcare: Practicing as if the natural environment really mattered. Altern. ther. health med. 2002; 8(5):76-83.

16. Souza MT, Silva MD, Carvalho R. Revisão integrativa: o que é e como fazer. Einstein. 2010; 8:102-106.

17. Bardin L. Análise de conteúdo. São Paulo: Edições $70 ; 2011$.

18. Muschel IJ. Pet therapy with terminal cancer patients. J. Soc. Casework. 1984; 65(8):451-458.

19. Roselló FT. Antropologia do cuidar. Rio de Janeiro: Petrópolis; 2009.

20. Wallis J, Lenon R. "Sow and grow", nature that nurtures: horticultural therapy as part of end-of-life care. BMJ Support Palliat Care. 2016; 6(suppl):1-25.

21. Erickson S. Restorative Garden Design: Enhancing wellness through healing, spaces. JAD, 2012; 2:89101.

22. Ulrich RS. How design impacts wellness. The Healthcare Forum Journal. 1992; 35(5):20-25.

23. Dalton C, Harrison JD. Designing an adaptive salutogenic care environment. In: Adaptive Architecture Conference, 1st International. London: Building Centre; 2011. 
24. Carlini S. Animal assisted therapy program: a co-therapy for palliative care. BMJ Support Palliat Care. 2016; 6(suppl1):25-26.

25. Chur-Hansen A, Zambrano SC, Crawford GB. Furry and feathered family members - A critical review of their role in palliative care. Am J Hosp Palliat Med. 2014; 31(6):672-677.

26. Coleman, CK. The experience of hospice caregivers providing animal-assisted therapy to hospice patients. [tese]. Minneapolis: Capella University; 2016. 214 p.

27. Leckie K, Pilgrem $\mathrm{L}$. There is more to gardening than meets the eye! BMJ Support Palliat Care. 2016; 6(suppl1):1-25.

28. López MLA. ¿̨La terapia asistida con animales influye de una manera positiva en los pacientes que padecen de una enfermedad terminal? [tese]. Medellín: Marymount School; 2013. 18 p.

29. MacDonald JM, Barrett D. Companion animals and well-being in palliative care nursing: a literature review. J. clin. nurs. 2016; 25(3-4):300-310.

30. Machnow Harju E, Vikberg F. Terapi på fyra ben: En litteraturöversikt om cancerpatienters upplevelse av djur i vården, [tese]. Sweden: Ersta Sköndal University College, Department of Health Care Sciences; 2015. 30 p.

31. Machnow Harju E, Vikberg F. Therapy on four legs A literary review about cancer patients experience of animals in care, 2015; p. 30.

32. Marsh P, Gartrell G, Egg G, et al. End-of-Life care in a community garden: Findings from a Participatory Action Research project in regional Australia. Health place. 2017; 45:110-116.

33. Schmitz A, Beermann M, MacKenzie CR, et al. Animal-assisted therapy at a University Centre for Palliative Medicine - A qualitative content analysis of patient records. BMC Palliat Care. 2017; 16(1):1-13.
34. Turnbach A. The Effect of Dog-Assisted Therapy on Cancer Patients in Hospice Care. J Psycol Res Propos. 2014; 4:7-12.

35. Gutiérrez G, Granados DR, Piar N. Interacciones humano-animal: características e implicaciones para el bienestar de los humanos. Rev. colomb. psicol. 2017; 16(1):163-184.

36. Queiroz RCFB. Eficácia da intervenção assistida por animais na autopercepção de saúde, autoestima, sintomas depressivos e qualidade de vida relacionada à saúde em idosos residentes em instituição de longa permanência. [dissertação]. Porto Alegre: Pontifícia Universidade Católica do Rio Grande do Sul; 2014. $116 \mathrm{p}$.

37. Fontana R, Wetler RMDC, Aquino RS, et al. Pathogenic bacteria dissemination by ants (Hymenoptera: Formicidae) in two hospitals in northeast Brazil. Neotrop. entomol. 2010; 39(4):655-663.

38. Engelman SR. Palliative care and use of animal-assisted therapy. Omega. 2013; 67(1-2):63-67.

39. Crippa A, Feijó ASG. Actividad asistida por animales como una alternativa complementaria para el tratamiento de los pacientes: labús queda por la evidencia científica. Rev. latinoam. bioét. 2014; 14:14-25.

40. Lima M, Sousa L. A influência positiva dos animais de ajuda social. Interações estud. pesqui. psicol. 2004; 4(6):156-174.

41. Costa EC. Animais de estimação: uma abordagem psico-sociológica da concepção dos idosos. [tese]. Fortaleza: Universidade Estadual do Ceará; 2006. 195 p.

42. Fournier AK, Geller ES, Fortney EE. Human-animal interaction in a prison setting: Impact on criminal behavior, treatment progress, and social skills. Behav Soc Issues. 2007; 169(1):89-105.

43. Rodrigues IG, Zago MMF. Cuidados Paliativos: Realidade ou utopia? Ciênc. cuid. saúde. 2009; 8(supl):136141. 
44. Murthy RK, Pandrangi V, Weber DJ. Animals in healthcare settings. In: Bearman G, et al. Infection Prevention. NewYork: Springer, Cham; 2018. p. 219233.

45. Matsumoto DY. Cuidados paliativos: conceitos, fundamentos e princípios. In: Academia Nacional de Cuidados Paliativos. Manual de cuidados paliativos. Rio de Janeiro: Diagraphic; 2009. p. 14-19.

46. Martín-Ortiz JD, Pérez MJS, Sierra JC. Evaluación de calidad de vida en pacientes con cáncer: una revisión. Rev. colomb. psicol. 2005; 14:34-45.

47. Siegel JM. Stressful life events and use of physician services among the elderly: The moderating role of pet ownership. J. pers. soc. psychol. 1990; 58(6):10811086.

48. Varas M. Terapia con mascotas. Medwave. 2010; 10(7):4617.

49. Kumasaka T, Masu H, Kataoka M, et al. Changes in Patient Mood through Animal-Assisted Activities in a Palliative Care Unit. Int Med J. 2012; 19(4):373-377.

50. Nebbe L. Nature Therapy. In: Handbook on Animal Assisted Therapy: Theoretical foundations and guidelines for practice. San Diego: Academic Press, 2000. p. $385-414$.

51. Academia Nacional de Cuidados Paliativos. Manual de cuidados paliativos. Rio de Janeiro: Diagraphic; 2011.

52. Kawakami CH, Nakano CK. Relato de experiência: terapia assistida por animais (TAA) - mais um recurso na comunicação entre paciente e enfermeiro. Brazilian Nursing Communication Symposium. 2002; An.8:1-7. [acesso em 2018 ago 4]. Disponível em: http://www. proceedings.scielo.br/scielo.php?script=sci_arttext \&pid=MSC0000000052002000100009\&lng=en\&n $\mathrm{rm}=\mathrm{iso} \& \operatorname{tng}=\mathrm{pt}$.

53. Sudnow D. Dead on arrival. Transaction. 1967; 5:3643.
54. Pessini L. Viver com dignidade a própria morte: reexame da contribuição da ética teológica no atual debate sobre a distanásia. [tese]. São Paulo: Pontifícia Faculdade de Teologia Nossa Senhora da Assunção; 2001.

55. Helgeson VS, Cohen S. Social support and adjustment to cancer: reconciling descriptive, correlational, and intervention research. Health psychol. 1996; 15(2):135-148.

56. Sadler CK. Design Guidelines for Effective Hospice Gardens Using Japanese Garden Principles. [tese]. New York: Faculty of Landscape Architecture, Suny College of Environmental Sciences and Forestry Syracuse; 2007.

57. Ulrich RS. Health benefits of gardens in hospitals. Paper for conference Plants for People, International Exhibition Floriade, 2002 [acesso em 2018 ago 2]. Disponivel em: https://www.researchgate.net/publication/252307449_Health_Benefits_of_Gardens_ in_Hospitals.

58. Phyllis T. Hospice care. Horticulture Sessions help hospice patients. [internet]. Farnham: Fundraising regulator; [s.d.]. [acesso em 2018 ago 4]. Disponível em: http://www.pth.org.uk/horticulture-sessions-at-pthc.

59. Instituto Brasileiro de Geografia e Estatística. População de animais de estimação no Brasil. Rio de Janeiro: IBGE; 2013.

60. Rocha RC. Visita de animal de estimação: proposta de atividade terapêutica assistida por animais a pacientes internados em hospital oncológico. [dissertação]. São Paulo: Pontifícia Universidade Católica de São Paulo; 2015.

Recebido em 22/01/2019

Aprovado em 10/05/2019

Conflito de interesses: inexistente

Suporte financeiro: não houve 